\title{
La actividad física recreativa disminuiría la mortalidad
}

\author{
Leisure time physical activity would reduce mortality
}

Moore SC y col. PLoS Med 2012;9(11):e1001335.

\begin{abstract}
Objetivos
Calcular los años de vida ganados (AVG) después de los 40 años según diferentes niveles de actividad física recreativa y según el Índice de Masa Corporal (IMC).

\section{Diseño, población y lugar}

Análisis de seis cohortes prospectivas (cinco estadounidenses, de las cuales dos incluían sólo mujeres, y una sueca) que habían participado de análisis previos de IMC y mortalidad en la National Cancer Institute Cohort Consortium, que incluían datos del nivel de actividad física realizada.

\section{Medición de resultados principales}

Se consideró actividad física recreativa a aquella de moderada a vigorosa intensidad, definida como la que implicaba un esfuerzo de 3 o más METS, el equivalente a caminar a paso rápido una hora por semana.

Los niveles de actividad física se dividieron en seis categorías equivalentes a las definidas por guías de actividad física estadounidenses ${ }^{1},{ }^{2}$ y de la OMS3: 0 MET-h/sem; 0,1 a 3,74 METh/sem; 3,75 a 7,4 MET-h/sem; 7,5 a 14,9 MET-h-sem; 15 a 22,4 MET-h/sem y >22,5 MET-h/sem (equivalentes a caminar a paso rápido por: 0; 1 a 74; 75 a 149; 150 a 299; 300 a 449 y $>450$ minutos por semana).
\end{abstract}

\section{Resultados principales}

Se analizaron 632.091 pacientes con una media de diez años de seguimiento. Ocurrieron 80.767 muertes entre los mayores de 40 años con IMC mayor a 18,5 .

Tabla 1: Mortalidad y AVG según nivel de actividad física tomando como referencia la no realización actividad física.

\begin{tabular}{|c|c|c|}
\hline $\begin{array}{c}\text { Actividad física } \\
\text { MET-h/sem }\end{array}$ & $\begin{array}{c}\text { Mortalidad }^{\dagger} \\
\text { HR ajustado* (IC 95\%) }\end{array}$ & Años de Vida Ganados (IC 95\%) \\
\hline 0,1 a 3,74 & $0,81(0,79$ a 0,83$)$ & $1,8(1,6$ a 2$)$ \\
\hline 3,75 a 7,4 & $0,76(0,74 \mathrm{a} 0,78)$ & $2,5(2,2$ a 2,7$)$ \\
\hline 7,5 a 14,9 & $0,68(0,66$ a 0,69$)$ & $3,4(3,2$ a 3,6$)$ \\
\hline
\end{tabular}

†Hazard ratio ajustado por sexo, consumo de alcohol, nivel educativo, estado civil, tabaquismo y comorbilidades (cáncer y enfermedad cardíaca).
A partir de los $15 \mathrm{MET}-\mathrm{h} / \mathrm{sem}$ en adelante los beneficios eran progresivamente mayores, pero con diferencias cada vez menos significativas.

No hubo diferencias significativas en el análisis conjunto de sexo y nivel de actividad física en cuanto al HR para mortalidad y los AVG. En cambio sí hubo un mayor beneficio a igual nivel de actividad física en quienes tenían mayores niveles de educación; en los ex tabaquistas comparado con los nunca habían fumado y con los tabaquistas activos y en quienes tenían una comorbilidad previa (cáncer o enfermedad cardiaca), aunque en este último grupo probablemente los que menos actividad realizaban fuesen los más enfermos.

El grupo de los más sanos (nunca fumaron y no tenían comorbilidades) fueron los que menos beneficios consiguieron con la actividad física, para una actividad de 22,5 MET-h/sem o mayor los AVG fueron de 2,8 (IC95\% 2,4 a 3,2), pero hay que tener en cuenta que este grupo era el que mayor expectativa de vida tenia independientemente del grado de actividad física.

Tabla 2: Años de vida perdidos (AVP) según IMC y actividad física.

\begin{tabular}{c|c|c|c|c|}
\hline \multirow{2}{*}{$\begin{array}{c}\text { Actividad física } \\
\text { MET-h/sem }\end{array}$} & $\begin{array}{c}\text { Normopeso } \\
\text { (IIMC 18,5 a 25) }\end{array}$ & $\begin{array}{c}\text { Sobrepeso } \\
\text { (IMC 25-30) }\end{array}$ & $\begin{array}{c}\text { Obesidad grado I } \\
\text { (IMC 30-35) }\end{array}$ & $\begin{array}{c}\text { Obesidad grado II } \\
\text { (IMC >35) }\end{array}$ \\
\hline $\mathbf{0}$ & 4,7 & 3,9 & 5 & 7,2 \\
\hline $\mathbf{0 , 1}$ a 7,4 & 2,4 & 1,8 & 3,2 & 6,2 \\
\hline$>\mathbf{7 , 5}$ & 0 (referencia) & 0 & 1,6 & 4,5 \\
\hline
\end{tabular}

†Hazard ratio ajustado por sexo, consumo de alcohol, nivel educativo, estado civil, tabaquismo y comorbilidades (cáncer y enfermedad cardíaca).

\section{Conclusión}

El incremento de los niveles de actividad física recreativa se asoció con un aumento de la esperanza de vida en los distintos niveles de actividad y grupos de IMC.

Fuente de financiamiento: Intramural Research Program del US National Institutes of Health (NIH) y la Division of Cancer Control and Population Sciences del National Cancer Institute.

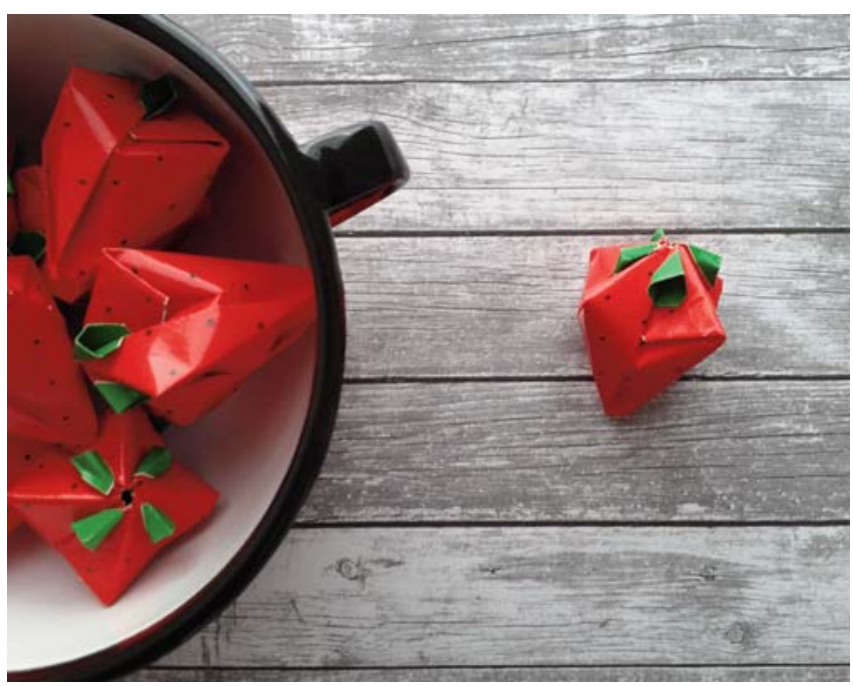

Foto y plegados de Claris Viviano (origami al alma) 


\section{Comentario}

Niveles muy por debajo de las recomendaciones de las guías de actividad física se asociarían a una disminución de la mortalidad y un aumento en la expectativa de vida. Estos beneficios existirían para cualquier nivel de IMC. Este último análisis conjunto de nivel de actividad física-IMC y expectativa de vida fue el aporte más novedoso de este estudio ya que no se encontraron en la bibliografía estudios de envergadura que hicieran un análisis semejante.

Los datos del análisis de nivel de actividad física-IMC y expectativa de vida mostraron que los AVP entre quienes tenían normopeso y no realizaban actividad física eran significativamente mayores $(4,7)$ a los que tenían sobrepeso y obesidad grado I pero realizaban actividad física, lo que refuerza aún más el valor de la actividad física incluso sobre el control del peso.

La dosis-respuesta de actividad física-mortalidad calculada en este estudio fue similar a la de estudios previos $^{2}$ y con una fuerza mayor debida a la extensa cantidad de participantes.

Las principales fortalezas del estudio fueron contar con una cohorte extensa, la amplitud de edades, categorías de actividad física bien definidas a través de cuestionarios validados y la posibilidad de estimar curvas de sobrevida en poblaciones previamente no determinadas.

Las limitaciones fueron la confiabilidad en los datos de nivel de actividad física ya que fueron auto-reportados, el estudio estuvo diseñado para actividad física recreativa (no tuvo en cuenta la actividad física realizada en el trabajo y en otras actividades no recreativas), confundidores no evaluados (otras comorbilidades, dieta, etc.), las cohortes analizadas eran heterogéneas y no se evaluó de la misma manera el nivel de actividad física (diferentes cuestionarios).

\section{Conclusiones del comentador}

A pesar de las limitaciones del estudio, su gran envergadura en cuanto a cantidad de participantes hace difícil pensar en conseguir mayores niveles de evidencia en cuanto a los objetivos planteados. Resulta revelador que niveles de actividad física incluso muy por debajo de los recomendados por las principales guías, tendrían beneficios significativos en términos de mortalidad y expectativa de vida, lo que podría tener un impacto altísimo a nivel poblacional. Por otro lado el estudio nos brinda información y datos fácilmente comunicables que nos pueden ayudar a reforzar el valor de la actividad física. Por último hay que destacar que estos resultados no cambian el nivel de evidencia, muchas veces cuestionado, de la consejería en el consultorio del médico de atención primaria sobre la actividad física. Sin embargo nos obliga a pensar en cuáles serian las mejores estrategias poblacionales para promover la realización de actividad física lo que tendría un impacto muy importante en términos de la salud pública.

Manuel Rodríguez Tablado [ Servicio de Medicina Familiar y Comunitaria del Hospital Italiano de Buenos Aires. manuel.rodriguez@ hospitalitaliano.org.ar ]

Rodríguez Tablado M. La actividad física recreativa disminuiría la mortalidad. Evid Actual Pract Ambul. 2015;18(3):78-79. Jul-Sep. Comentado de: Moore SC, y col. Leisure Time Physical Activity of Moderate to Vigorous Intensity and Mortality: A Large Pooled Cohort Analysis. PLoS Med 2012; 9(11): e1001335.PMID: 23139642.

\section{Referencias}

1. US Department of Health and Human Services (2008) 2008 physical activity guidelines for Americans. Washington (District of Columbia): US Department of Health and Human Services

2. Physical Activity Guidelines Advisory Committee (2008) Physical Activity Guidelines Advisory Committee report, 2008. Washington (District of Columbia): US Department of Health and Human Services.

3. World Health Organization (2010) Global recommendations on physical activity for health. Geneva: World Health Organization.

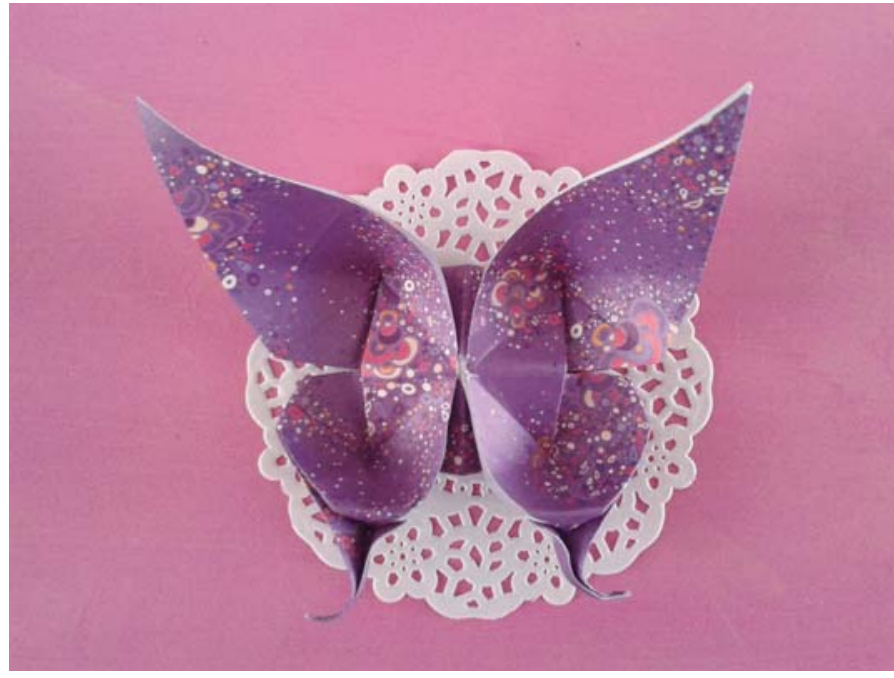

Foto y plegados de Claris Viviano (origami al alma) 